\title{
Adhesives Manufacturer Optimises Logistics
}

The adhesives company Jowat has significantly improved the performance of its warehouse and picking processes using a mobile data entry system supplied by AISCI Ident. This enables a much larger volume of its products to be processed in a shorter time and is accompanied by a long-term increase in process reliability.

A round two years ago, Jowat brought into operation a new logistics centre at its headquarters in the German town of Detmold, which resulted in a considerable increase in its warehouse, picking and goods inwards capacity. The new centre can house up to 11,000 pallets, which is the equivalent of around 350 truckloads. The total investment in the centre amounted to approximately EUR 10 million.

With the opening of the new building, the company also modernised its logistics processes. Jowat relies on the expertise of the automated identification systems company AISCI Ident for its mobile data entry hardware. "This shortens the time needed for our processes and enables us to make our workflows much more customer-specific," explains Samir Youssef, who has overall responsibility for the IT and communication technology aspects of the project at Jowat. "The increase in efficiency and reduction in communication in our internal processes have allowed us to handle larger volumes." The new solution has also made the processes much more reliable.

\section{High levels of transparency}

"We have already supplied mobile data entry devices for Jowat's produc- tion facilities and warehouses in the past," explains Sascha Radovic, the project manager at AISCI Ident. "As part of the modernisation process that accompanied the construction of the new logistics centre, we were able to support Jowat with the introduction of fixed data entry terminals mounted on the forklift trucks and mobile handheld terminals for use in picking processes." AISCI Ident also recommended which type of hardware should be used on the different sizes of truck, in order to ensure that the logistics workflows were clear and transparent. ads-tec, a hardware manufacturer from Leinfeld in Germany, supplied the forklift terminals.

The next important question concerned the choice of handheld scanners that would provide the best possible support for the data entry and processing tasks. Ultimately, Jowat decided on Powerscan Extra Long Range scanners from Datalogic, which can read data from a distance of up to 10 metres. This allows barcodes to be scanned from the forklift and sent to the server via a Telnet client connection. Server windows are available on the forklift terminals, enabling the data to be made available immediately in the SAP system. As part of the modernisation process, AISCI Ident installed a WLAN in the warehouse, upgraded the existing access points and selectively improved the performance of the existing infrastructure.

\section{Mobile printer solution}

Another of Jowat's key requirements was a mobile printer solution to enable forklift truck drivers and picking staff to print small labels in their own work areas. "We have introduced a special vehicle mount for the Toshiba two-inch printer, which takes its power supply from the truck," explains AISCI project manager Sascha Radovic. The process is as follows. When a new order arrives in the picking department, the barrels or pallets are labelled with a barcode containing all the necessary data relating to the customer and the order. The SAP system issues a print command and the label is printed on the mobile printer via the WLAN. The picker attaches the label to the barrel or pallet.

Jowat is very pleased with the system as it currently stands. "In future, we would like to work together with AISCI in other areas and we are currently considering introducing a system to enter operating data using barcodes," says Jowat project manager Samir Youssef. -

Author: Thomas Wöhrle, technical journalist, Karlsruhe

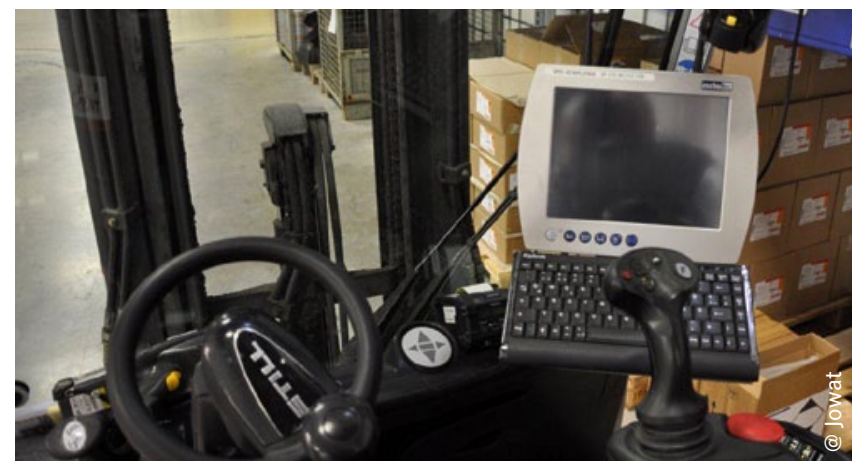

Jowat has installed the latest data entry terminals on its forklift trucks.

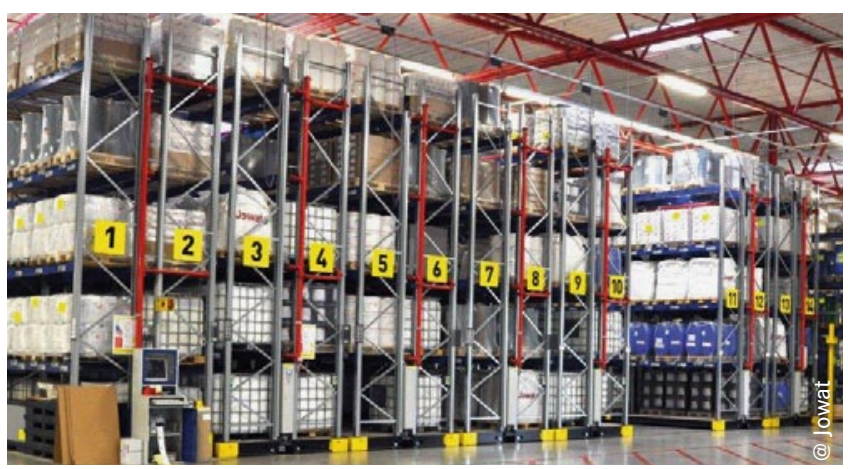

The construction of the new logistics centre also enabled Jowat to update its logistics workflows. 